\title{
The Wound Healing Effect of Nanoclay, Collagen, and Tadalafil in Diabetic Rats: An In Vivo Study
}

\author{
Allahyar Noori Ordeghan, ${ }^{1}$ Danial Khayatan ${ }^{(D},{ }^{2}$ Mohammad Reza Saki, ${ }^{1}$ Mostafa Alam, ${ }^{3}$ \\ Kamyar Abbasi, ${ }^{4}$ Hossein Shirvani, ${ }^{1}$ Mohsen Yazdanian $\mathbb{D D}^{5,6}$ Reza Sayyad Soufdoost, ${ }^{7}$ \\ Haidar Taimouri Raad, ${ }^{5,6}$ Ali Karami, ${ }^{5,6}$ and Hamid Tebyaniyan $\mathbb{D}^{8}$ \\ ${ }^{1}$ Exercise Physiology Research Center, Life Style Institute, Baqiyatallah University of Medical Sciences, Tehran, Iran \\ ${ }^{2}$ Faculty of Pharmacy, Tehran Medical Sciences, Islamic Azad University, Tehran, Iran \\ ${ }^{3}$ Department of Oral and Maxillofacial Surgery, School of Dentistry, Shahid Beheshti University of Medical Sciences, Tehran, Iran \\ ${ }^{4}$ Department of Prosthodontics, School of Dentistry, Shahid Beheshti University of Medical Sciences, Tehran, Iran \\ ${ }^{5}$ Research Center for Prevention of Oral and Dental Diseases, Baqiyatallah University of Medical Sciences, Tehran, Iran \\ ${ }^{6}$ School of Dentistry, Baqiyatallah University of Medical Sciences, Tehran, Iran \\ ${ }^{7}$ Imam Khomeini Clinic of Dentistry, Tehran, Iran \\ ${ }^{8}$ Islamic Azad University, Science and Research Branch, Tehran, Iran
}

Correspondence should be addressed to Mohsen Yazdanian; myazdaniandr@gmail.com and Hamid Tebyaniyan; tebyan.hamid@yahoo.com

Received 23 September 2021; Revised 21 November 2021; Accepted 2 January 2022; Published 15 January 2022

Academic Editor: Ravichandran M

Copyright (c) 2022 Allahyar Noori Ordeghan et al. This is an open access article distributed under the Creative Commons Attribution License, which permits unrestricted use, distribution, and reproduction in any medium, provided the original work is properly cited.

\begin{abstract}
The diabetic wound is the most challenging one to manage, which is associated with microvascular and macrovascular dysfunction, and novel strategies such as using hydrogels demonstrate their promising prospect in treatment and management approaches of the diabetic wound. This study aimed to investigate the effect of collagen/nanoclay/tadalafil hydrogel on wound healing in diabetic rats under HIIT exercise. Hydrogel was synthesized, and then biocompatibility and antibacterial tests were performed. The therapeutic effect of collagen/ nanoclay/tadalafil hydrogel was assessed after induction of diabetes in the rat model, and wound healing was evaluated with macroscopic and microscopic tests. The result of the MTT test showed no significant cytotoxicity of collagen/nanoclay/tadalafil hydrogel. Furthermore, the inhibitory effect of hydrogel was detected on E. coli and S. aureus. The macroscopic results demonstrated that the wound contraction was considerable in the hydrogel/HIIT exercise and hydrogel groups compared with the HIIT exercise and control groups during 21 days. The microscopic results showed that the presence of fibroblasts, the amount of collagen, the epidermis density, and the formation of hair follicles were increased in the hydrogel/HIIT exercise group compared with other groups in the diabetic rate model. It can be concluded that collagen/nanoclay/tadalafil hydrogel with HIIT exercise could accelerate diabetic wound healing and can be an appropriate candidate for skin regeneration in medical applications.
\end{abstract}

\section{Introduction}

Diabetes has also known as a comprehensive metabolic disorder that impacts about 340 million people worldwide. Also, diabetic wounds have been developed in approximately $20 \%$ of people with diabetes. Hyperglycemia causes the most microvascular and macrovascular dysfunctions that entangle the process of wound healing. One of the common wound types is a diabetic foot ulcer that is widespread among all age groups and is more common in individuals more than 65 years with an incidence of about $3 \%$ and a prevalence of near $1 \%$ of the world population. About 2.5 to 15 percent of the world's annual health expenditure is spent on diabetes, and diabetic ulcers account for a large proportion of it. The World Health Organization reports that diabetes will be the 7 th leading cause of death in 
$2030[1,2]$. Diabetic wound treatments involved pressure off-loading, debridement of wound, infection management with topical antiseptics and topical antibiotics, and revascularization, which are the main principles of diabetic wound therapeutic approaches. There are various approaches to debridement such as surgical procedures, wet to dry dressing, biosurgery, preparations of enzymes, hydrogels, and dextranomer polysaccharide paste or polysaccharide beads. When wound dressings change the wound from moist to dry, they supply nonselective debridement, thus cleaning the wound with necrotic tissue removal. It is suggested that wounds be evaluated at every dressing change. Moisture-retentive dressings involving foams, hydrocolloids, hydrogels, films, hydrofibers, and alginates are helpful in different clinical settings [3].

HIIT (High-Intensity Interval Training) is based on the principle of exercise intensity that is a more sufficient modifier constituent of exercise causing adjustment in the human body system containing nervous [4], cardiovascular [5], and skeletal muscle [6] system. HIIT has been known as a time-efficient exercise method that is well described as short durations of high-intensity exercise (more than 80-85\% peak oxygen uptake), alternating with active or passive rest periods [7]. Recently, HIIT has received great consideration for its impact on improving superior vascular function improvements compared to continuous training with moderate intensity in healthy people and several clinical patients containing metabolic syndrome, heart failure, and obesity [8]. Despite HIIT improvement effects on vascular function in a patient with systemic limited cutaneous sclerosis [9], its special effects on skin remain untested. New therapeutic approaches such as extracellular matrix proteins, bioengineered skin substitutes, negative pressure wound therapy, and growth factors have been indicated as adjunctive treatments for wounds [10]. Different biomaterials are also used in clinical wound therapy. For example, oxidized regenerated collagen/cellulose was primarily indicated to facilitate wound closure in a diabetic model in mice and acute wounds in rats. Different biomaterials are being produced in the regeneration of tissue to emerge as scaffolds where the cells are implanted or as vehicles for specified drug release. These scaffolds illustrate the source of tissue topology and provide cues that are topological and promote tissue regeneration. Many synthetic or natural polymers, also named hydrogels, are being progressed and investigated in preclinical researches. Biodegradable films are made of hydrogels or hydrocolloids and thus, by regeneration, can be integrated into the wound of patients [11]. One of the phosphodiesterase type 5 (PDE5) inhibitors is tadalafil which reduces cGMP (cyclic guanosine monophosphate) destruction and afterward increases the NO (nitric oxide) vasodilation effect. Usually, tadalafil has been utilized for controlling pulmonary arterial hypertension and erectile dysfunction through inhibition of NO collapse-driven cGMP in platelets aggregation and vascular smooth muscle, which impact vasodilation of peripheral blood vessels. Tadalafil also can preserve tissues, for instance, lung, skin, and brain against ischemia [12]. Furthermore, some animal studies have demonstrated that PDE5 inhibitors promote wound healing [13]. Inhibitors of PDE5 also upregulate iNOS (inducible nitric oxide synthase) by the pathway of NOcGMP and develop the wound and fractures healing procedure [14]. Till now, there is no reported side effect in animal models when tadalafil is used in the wound healing process. Nanocomposite hydrogels of nanoclay would be provided easily and conveniently and demonstrated mechanical properties, biocompatibility, good swelling properties, and optical properties. Also, findings demonstrated that hydrogels composed with nanoclay could promote the connection of dermal fibroblasts of human on the surface of the hydrogels $[15,16]$. Collagen is responsible for supporting the integrity of the cells and tissue structures that are the most protein and exist naturally. Skin collagen has been synthesized by myofibroblasts and fibroblasts that are arranged in the fibril forms of cutaneous tissue, bone, and tendons, which consistently undergo tensile and shear stresses. Type I collagen is found in the tendons, fasciae, and dermis and is the most important component in scar tissue. Collagen is also utilized in synthesizing skin substitutes that are also present in beaded structures, fibril surfaces, or transmembrane proteins composition [17]. Despite the fact that collagen, nanoclay, and tadalafil have been investigated individually in wound healing experimental studies, no evaluation exists to evaluate the effect of collagen/nanoclay/tadalafil combination on wound healing in vivo. This study aimed to assess the effect of collagen/ nanoclay/tadalafil hydrogel on complete wound healing in type I diabetic rats under HIIT exercise.

\section{Materials and Methods}

2.1. Materials. Hydrochloric acid and tadalafil were purchased from Sigma-Aldrich (Germany). The solvents, chloroform, and methanol were obtained from SigmaAldrich (USA) and Carlo Erba (Italy). Collagen type I was obtained from Medzist and TAB Nano (Iran). The nanoclay was purchased from Southern Clay Products, Inc. Staphylococcus aureus (ATCC 25923) and Escherichia coli (ATCC 25922) strains and human embryonic kidney cells (HEK293) were gifted. MTT Kit was purchased from Bio Idea (Iran). DMSO (dimethyl sulfoxide) was purchased from SigmaAldrich (Germany). FBS (fetal bovine serum), DMEM (Dulbecco's modified Eagle's medium), PBS (phosphatebuffered saline), trypsin, ascorbic acid 3-phosphate, alkaline phosphatase kit, dexamethasone, beta-glycerol, and antistreptomycin were obtained from Gibco.

\subsection{Synthesis of Hydrogel. To acquire collagen/nanoclay/} tadalafil hydrogel, $10 \% \mathrm{w} / \mathrm{v}$ collagen solution was provided in $1 \% \mathrm{v} / \mathrm{v}$ acid acetic solution $(\mathrm{pH}=2)$ and stirred well ( $40 \mathrm{rpm}, 4^{\circ} \mathrm{C}, 24$ hours). Then, $\mathrm{pH}$ was adjusted to 7 (by adding $0.5 \mathrm{M}$ of $\mathrm{NaOH}$ under stirring for neutralizing the hydrogel). After preparation of the solution, tadalafil powder $(30 \mathrm{mg} / \mathrm{ml})$ was added to the solution of collagen while stirring $\left(4^{\circ} \mathrm{C}, 2\right.$ hours). The obtained nanoclay $(170 \mathrm{mg} / \mathrm{ml})$ was centrifuged, washed (three times with DW and 
lyophilized), and added progressively to the solution. The acquired homogeneous suspension was kept at $4^{\circ} \mathrm{C}$.

2.3. Cell Culture. HEK293 were cultured in DMEM involving $10 \% \mathrm{FBS}$, streptomycin sulfate $(10 \mathrm{mg} / \mathrm{ml})$, and penicillin $\mathrm{G}$ sodium (10 units $/ \mathrm{ml}$ ) in a humidified incubator $\left(5 \% \mathrm{CO}_{2}, 90 \%\right.$ humidity, $\left.37^{\circ} \mathrm{C}\right)$. The culture medium was refreshed every 48 hours to achieve the confluence of $80 \%$. The second passage of cells was utilized for the next step.

2.4. Biocompatibility Test. The MTT (methyl thiazolyl tetrazolium) test was performed straightforwardly according to the ISO 10993-5 standards. The cell culture plate serves as the negative control (without cytotoxicity). MTT assay was conducted to investigate the hydrogel cytotoxicity. For the aim, 20,000 HEK 293 cells were added to the culture medium $(50 \mu \mathrm{L})$, including serum, and then cultured on the hydrogel. After 3 hours, the culture medium was added to each well. The culture medium was removed, and then, MTT solution $(100 \mu \mathrm{L}, 0.5 \mathrm{mg} / \mathrm{mL})$ was added to each well. The supernatant solution was eliminated after 4 hours. Afterward, DMSO $(100 \mu \mathrm{l})$ was added to dissolve the formed purple formazan crystals. The solution optical density was read at 570 nanometers wavelength in an ELISA reader (Convergent ELReader 96X). Outcomes were displayed as a percentage $(100 \%=$ control value $)$, and the assays were conducted in triplicate to obtain accurate results. The viability of cell was measured using the following equation:

$$
\text { cell viability }(\%)=\frac{\text { value of therapy sample }}{\text { value of ample control }} \times 100 \text {. }
$$

2.5. Antibacterial Analysis. Staphylococcus aureus (Grampositive bacteria) and Escherichia coli (Gram-negative bacteria) were utilized to evaluate the antibacterial activity of the collagen/nanoclay/tadalafil hydrogel. LB agar (Luria-Bertani agar) and LB broth (Luria-Bertani broth) were conducted as nutrient sources for the cell culture medium. Staphylococcus aureus and Escherichia coli were added to the LB broth and incubated in a shaking incubator (24 hours, 5\% $\mathrm{CO}_{2}, 37^{\circ} \mathrm{C}$ ). The bacteria concentration in the culture medium was $10^{6} \mathrm{CFU} \mathrm{mL}^{-1}$ (colony-forming units per milliliter). The hydrogel was incubated in LB broth (24 hours, $37^{\circ} \mathrm{C}$ ). Afterward, the viable bacteria number was calculated to investigate the antibacterial activity. Also, the inhibition zone was evaluated, and ciprofloxacin was performed as the standard antibiotic disc (control).

2.6. In Vivo Wound Healing in Diabetic Rats. To investigate the ability of collagen/nanoclay/tadalafil hydrogel in fullthickness wound healing, 36 adults male rats (white Wistar rats, $165 \pm 15$, aged 6-8 weeks) were utilized. Also, rats were bought from Animal Research. Furthermore, rats were divided into 4 groups ( $n=3$ for each time $(7,14$, and 21 days)), control and test groups (hydrogel, hydrogel with HIIT exercise, and only HIIT exercise) accidentally. The rats were housed in wire-topped cages with sterile rice husk as bed material and kept at standard conditions $\left(22-25^{\circ} \mathrm{C}\right.$, 65-75\% humidity, 12 hours of light/dark cycles, fed with pellets and water ad libitum). All animal tests were examined based on the guidelines correlated with the Care and Use of Laboratory Animals. For induction of experimental type I diabetes in overnight fasted rats, single intraperitoneal injections of STZ (streptozotocin) and nicotinamide were used. First, nicotinamide was dissolved in sterile water and injected $(110 \mathrm{mg} / \mathrm{kg})$. After 15 minutes, STZ, dissolved in cold citrate buffer $(\mathrm{pH}=4.5,0.1 \mathrm{M})$, was injected ( $50 \mathrm{mg} / \mathrm{kg}$ ). After 72 hours, diabetes was confirmed by measuring glucose of the tail vein blood using a glucometer (Bayer Contour TS Blood Glucose Monitor). After three weeks, rats with blood glucose levels $>250 \mathrm{mg} / \mathrm{dL}$ were examined as diabetic and used for the in vivo studies [18].

2.7. Wound Model. Anesthesia is induced by IM (intramuscular) injection of ketamine $(50 \mathrm{mg} / \mathrm{kg}$, ketamine $10 \%)$ and xylazine $(10 \mathrm{mg} / \mathrm{kg}, 2 \%)$. Dorsal skin was shaved and then sanitized using ethanol. Afterward, incisions $(8 \mathrm{~mm})$ were created by the circular punch. All diabetic rats were tested in four groups: Group 1: control (untreated), Group 2: HIIT exercise, Group 3: hydrogel, and Group 4: hydrogel/ HIIT exercise during 21 days.

2.8. High-Intensity Interval Training Program. HIIT program was considered as three times a week on a treadmill [19]. To summarize, the program of HIIT contained a warm-up (5 minutes) and cooling section (a $40 \%$ double-speed), and the major part comprised one-minute intense running (5-7 sessions, at $80-90 \%$ of the maximum intensity of running speed test) and in rest rotations with 55\% with zero slopes of the treadmill. The HIIT program details are presented in Table 1. The MERT is utilized in the first week before the beginning of the training protocol and at the end of the three-week program for finding out the maximum training intensity and capacity [20].

2.9. Macroscopic Evaluation. For measuring the contraction of the wound, the images were taken from wounds on days 7 , 14 , and 21 . The average wound area was measured using ImageJ software (NIH, Maryland), and the percentage of wound contraction was calculated using the following method: 
TABLE 1: High-intensity interval training protocol.

\begin{tabular}{|c|c|c|c|c|c|}
\hline & \multirow{2}{*}{ Warm-up } & \multicolumn{2}{|c|}{ Main seasons } & \multirow{2}{*}{ Cold own } & \multirow{2}{*}{ Total time $(\mathrm{min})$} \\
\hline & & Intense periods & Rest & & \\
\hline Week 1 & & $5 \times 1 \min (80 \%$ MERT $)$ & $5 \times 1 \min (55 \%$ MERT $)$ & & 20 \\
\hline Week 2 & $5 \min (40 \%$ MERT $)$ & $6 \times 1 \min (80 \%$ MERT $)$ & $6 \times 1 \min (55 \%$ MERT $)$ & $5 \min (40 \%$ MERT $)$ & 22 \\
\hline Week 3 & & $7 \times 1 \min (80 \%$ MERT $)$ & $7 \times 1 \min (55 \%$ MERT $)$ & & 24 \\
\hline
\end{tabular}

MERT: maximum running sprint test; min: minute.

wound contraction $(\%)=\frac{\text { initial wound size }- \text { specific day wound size }}{\text { initial wound size }} \times 100$.

2.10. Microscopic Evaluation. Tissue sampling was analyzed on days 7, 14, and 21 for each group. The thick part of the skin around the wound was dissected, disinfected with normal saline solution, placed in formalin solution $(10 \%$, $24 \mathrm{~h}$ ), and fixed in paraffin using a tissue processor. The tissue sections $(5 \mu \mathrm{m})$ were sliced to the wound surface perpendicularly, embedded on a slide, and stained with Hematoxylin and Eosin (H\&E) and Trichrome-Masson reagents for histopathological studies. The wound healing of samples was investigated by a light microscope (Leica Microsystems, Wetzlar, Germany).

2.11. Statistical Analysis. Data were analyzed using SPSS (Kruskal-Wallis (nonparametric)), and Mann-Whitney test was used for comparing the groups. The $p$ value was considered statistically significant $(p<0.05)$.

\section{Results}

3.1. In Vitro Biocompatibility of the Hydrogels. Biocompatibility was studied by culturing HEK 293 cells on hydrogels and control (tissue culture plate) for 24,48 , and $72 \mathrm{~h}$. The developed hydrogel with collagen/nanoclay/ tadalafil was prepared and investigated for wound healing in diabetic wounds with and without HIIT exercise. The MTT test showed no significant cytotoxicity, and the percentage of cell viability was more than $90 \%$ for 24,48 , and $72 \mathrm{~h}$ (Figure 1).

3.2. Antibacterial Analysis. The antibacterial activity of the collagen/nanoclay/tadalafil hydrogel was investigated by two bacterial species, Staphylococcus aureus (Gram-positive bacteria) and Escherichia coli (Gram-negative bacteria). The quantitative results of antibacterial activity showed that CFU (colony-forming unit) of S. aureus was $140 \times 10^{6}$ and CFU of E. coli was $95 \times 10^{6}$ in the collagen/nanoclay/tadalafil hydrogel, while the CFU of S. aureus and E. coli was $260 \times 10^{6}$ and $240 \times 10^{6}$ in the control group, individually. However, the number of bacteria was reduced in the collagen/nanoclay/tadalafil hydrogel. Therefore, this result revealed that the hydrogel might conduct an antibacterial effect on the healing process of the wound. Also, the size of hydrogels inhibition zones was estimated against $E$. coli and $S$. aureus as the principal species for infections of the wound as demonstrated in Table 2; ciprofloxacin standard disc was selected as the control group in comparison with the collagen/nanoclay/tadalafil hydrogel inhibitory action. Both $E$. coli and $S$. aureus were susceptible to the ciprofloxacin discs (the control group). The collagen/nanoclay/tadalafil hydrogel displayed an inhibition zone in comparison with the standard antibiotic disc because of the presence of nanoclay in the hydrogel.

3.3. Macroscopic Evaluation. Results of macroscopic evaluation showed significant changes in the percentage of wound healing of the HIIT exercise, hydrogel, and hydrogel/ HIIT exercise groups compared with the control group in 14 and 21 days (Figure 2). The contraction rate of the wound in the hydrogel and hydrogel/HIIT exercise groups was more than that in the control group. Also, the percentage of wound contraction rate was remarkably increased in the hydrogel/HIIT exercise group, although the rate of wound contraction was enhanced in each group during 21 days. The healing rate of hydrogel was more than that of the HIIT exercise group, and the healing rate of hydrogel/HIIT exercise was more than that of the control group (Figure 3). These findings indicated the ability of hydrogel and HIIT exercise in the regeneration of diabetic wounds. The evaluation of diabetic wound healing rate was measured by ImageJ in this study. The results displayed that diabetic wound healing rate in 21 days of study with a hydrogel which is loaded with collagen has changed significantly (Table 3). The wound contraction rate in the hydrogel/HIIT exercise group was more than that in the HIIT exercise and hydrogel groups on days 14 and 21, respectively. Moreover, the contraction rate of the hydrogel group was much higher than that of the HIIT exercise and control groups. The results showed that hydrogel could heal diabetic wounds with HIIT exercise.

3.4. Microscopic Evaluation. Histopathological samples were used in microscopic analyses that estimate the wound healing in the granulation tissues collected during 21 days. Tissue sections were stained with H\&E and Masson's trichrome stain. Early stages of cellular activities and wound healing through H\&E staining were observed (Figures 4 and 5). Deposition and formation of collagen were evaluated using Masson's trichrome staining qualitatively (Figures 6 and 7 ). In the control group, the microscopic evaluation showed no substantial changes in the rate of wound healing 


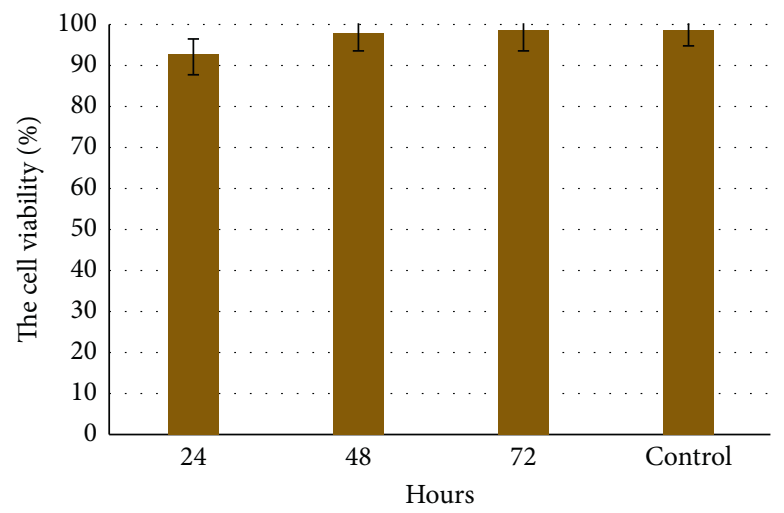

FIGURE 1: The cell viability study of the collagen/nanoclay/tadalafil hydrogel on HEK 293 cells for 24, 48, and $72 \mathrm{~h}$ three independent tests was conducted $(p<0.05)$.

TABLE 2: The antibacterial activity of collagen/nanoclay/tadalafil hydrogel against E. coli and S. aureus.

\begin{tabular}{lcc}
\hline Samples & & Inhibition zone \\
& Staphylococcus aureus & Escherichia coli \\
\hline Collagen/nanoclay/tadalafil hydrogel & 10.2 & 14.5 \\
Ciprofloxacin disc & 26.7 & 27.3 \\
\hline
\end{tabular}

All samples were tested three times in independent experiments $(p<0.05)$.

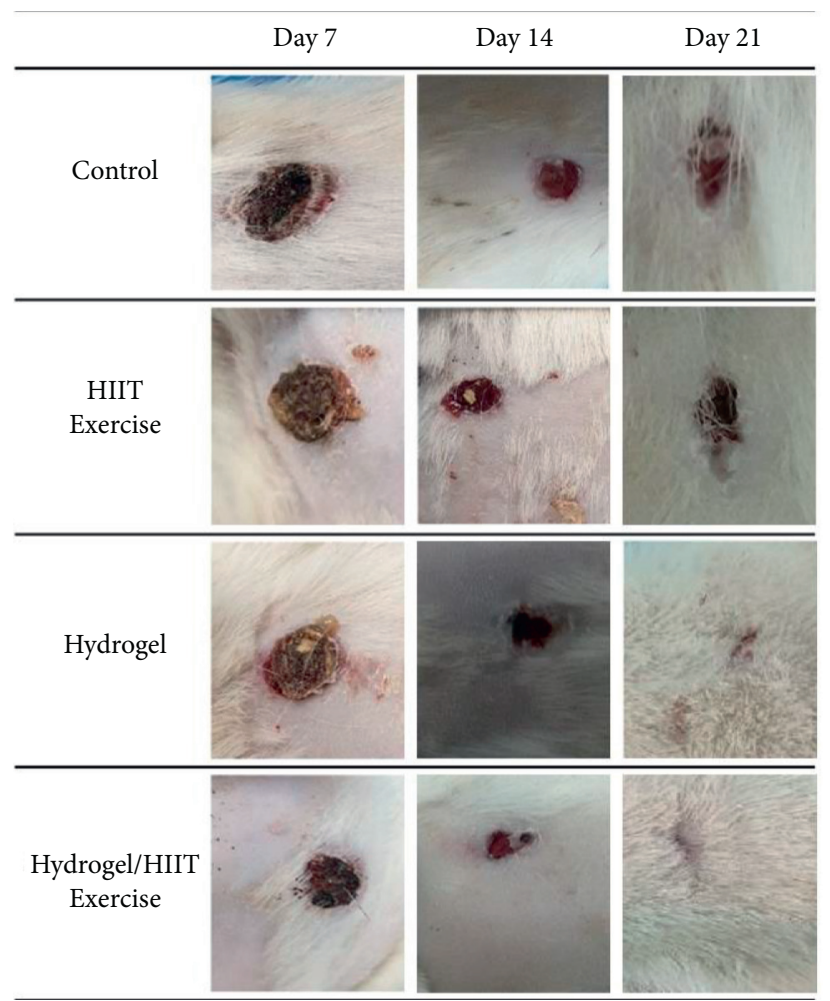

FIGURE 2: Macroscopic evaluation of the control, HIIT exercise, hydrogel, and hydrogel/HIIT exercise groups on days 7, 14 , and 21.

from margin to the center on day 7 , the defect was covered by a very thin layer of connective tissue, and the wound healing was higher on day 14 compared to day 7 . Wound healing from the margin to the center of the defect was detected with H\&E and Masson's trichrome staining. On day 21 , the rate of healing and infiltration of cells into the center of the defect as well as the thickness of the three layers was significant compared to day 14 (Figures 4(a) and 6(a)). 


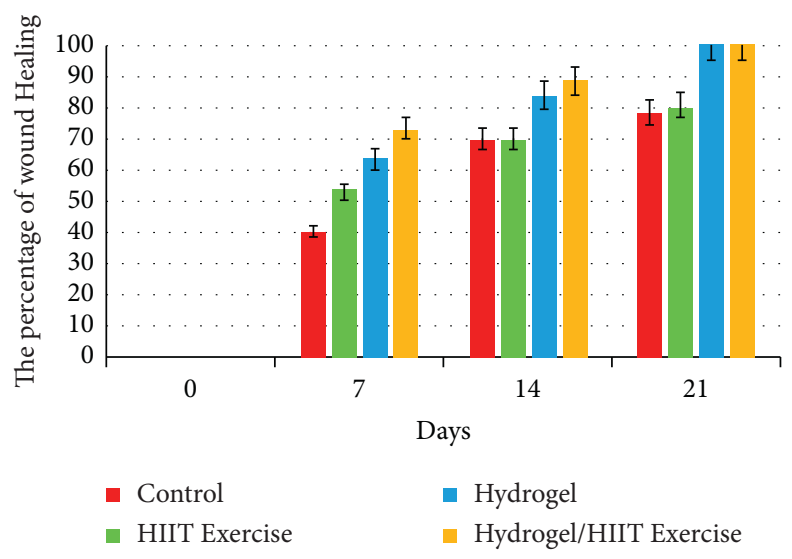

FIGURE 3: The rate of wound contraction in the control, HIIT exercise, hydrogel, and hydrogel/HIIT exercise groups on days 7, 14 , and 21.

TABLE 3: The wound contraction rate (millimeter) in $0,7,14$, and 21 days.

\begin{tabular}{lcccc}
\hline Groups & Day 0 & Day 7 & Day 14 & Day 21 \\
\hline Control & 8 & 4.77 & 2.44 & 1.7 \\
HIIT exercise & 8 & 3.76 & 2.4 & 1.55 \\
Hydrogel & 8 & 2.9 & 1.3 & 0 \\
Hydrogel/HIIT exercise & 8 & 2.11 & 0.9 & 0 \\
\hline
\end{tabular}

All samples were tested three times in independent experiments $(p<0.05)$.

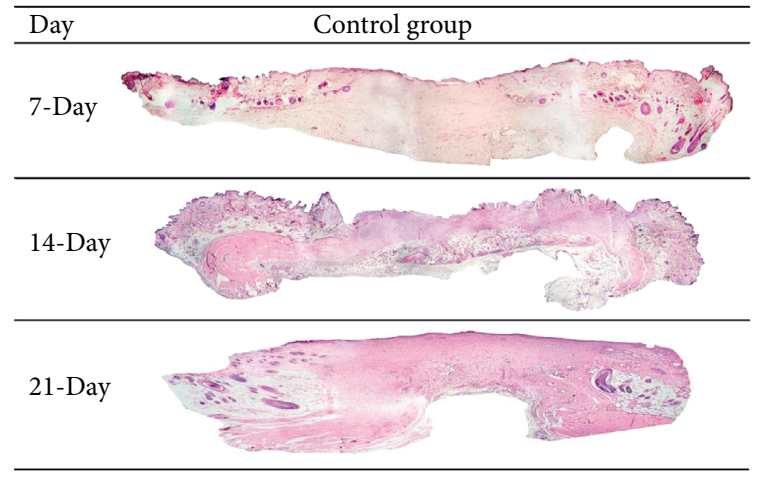

(a)

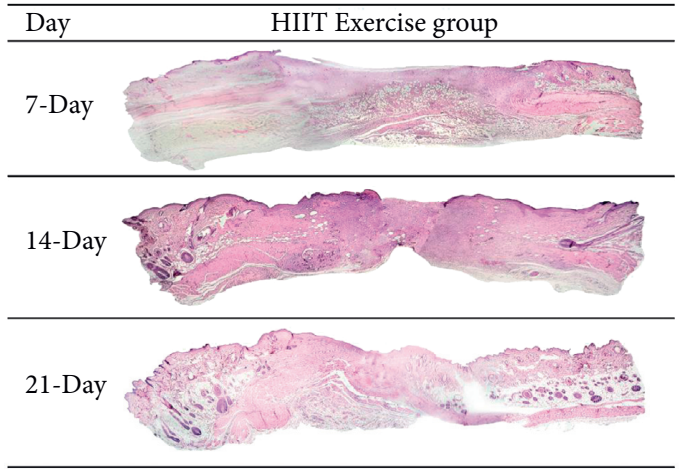

(b)

FIgURE 4: Histological analysis of control $(\mathrm{a}: \times 40)$ and HIIT exercise $(\mathrm{b}: \times 40)$ with H\&E staining.

In the HIIT exercise group, cell infiltration was observed in the center of the defect on day 7 , and a small amount of healing and high density of connective tissue layer was detected. On day 14, the formation of skin layers, the rate of healing, and infiltration of cells were observed, and wound healing was increased compared with day 7 . The amount of healing and formation of three layers of skin was determined on day 21. The formation of a relatively regular layer of collagen and the presence of hair follicles were observed (Figures 4(b) and 6(b)).

In the hydrogel group, there was no healing from margin to center during 7 days, but infiltration of cells into the center area of defect and forming of connective tissue were detected. The amount of regeneration and infiltration of cells from to the center of the defect was observed on day 14, and the formation of an irregular layer of collagen and hair follicles was observed in the margin of the defect. The amount of healing and infiltration of cells from the margin to the center of the defect was observed on day 14, and the formation of an irregular layer of collagen and hair follicles was detected in the margin of the defect. On day 21, this amount of three-layer healing and the presence of hair follicles were remarkable in the defect site. In addition, regular collagen was observed in the margin and defect site, and this amount of healing from the margin to the center was significant compared to days 7 and 14 (Figures 5(a) and $7(\mathrm{a}))$.

In the hydrogel/HIIT exercise group, the rate of connective tissue formation was very high in the defect site, and the infiltration of cells and wound healing was relatively 


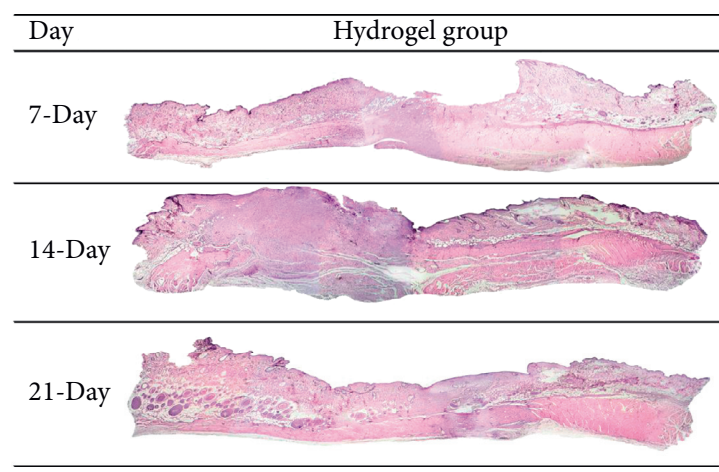

(a)

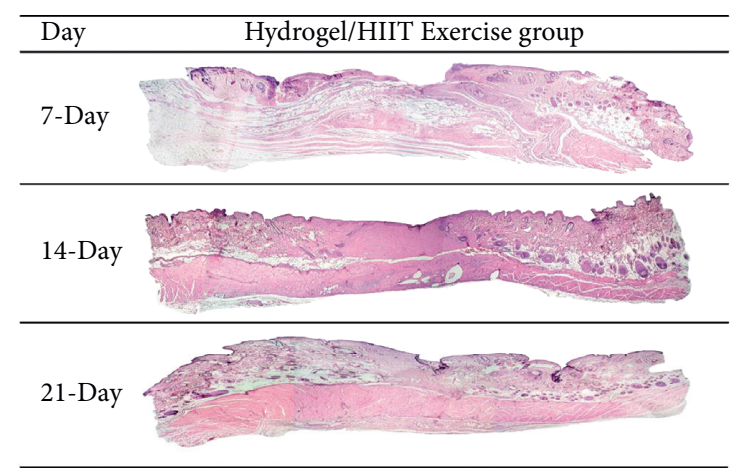

(b)

FIGURE 5: Histological analysis of hydrogel $(\mathrm{a}: \times 40)$ and hydrogel/HIIT exercise $(\mathrm{b}: \times 40)$ with H\&E staining.

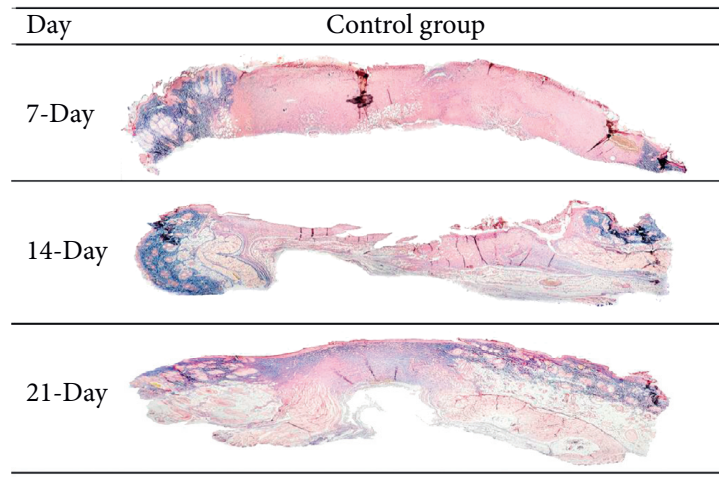

(a)

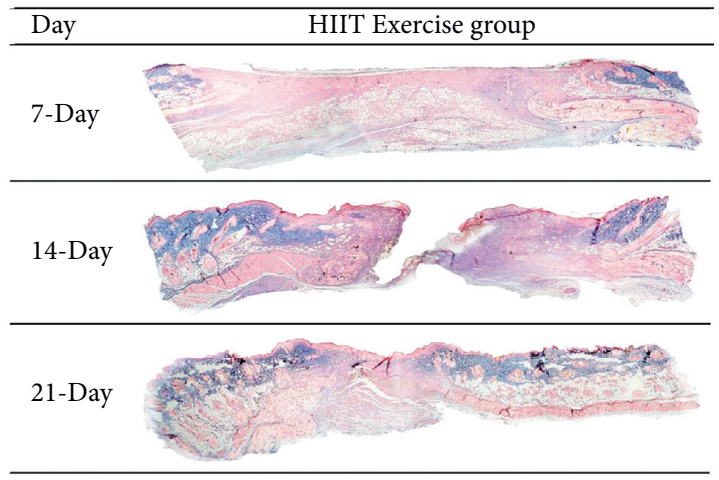

(b)

FIgURE 6: Histological analysis of control $(\mathrm{a}: \times 40)$ and HIIT exercise $(\mathrm{b}: \times 40)$ with Masson's trichrome staining.

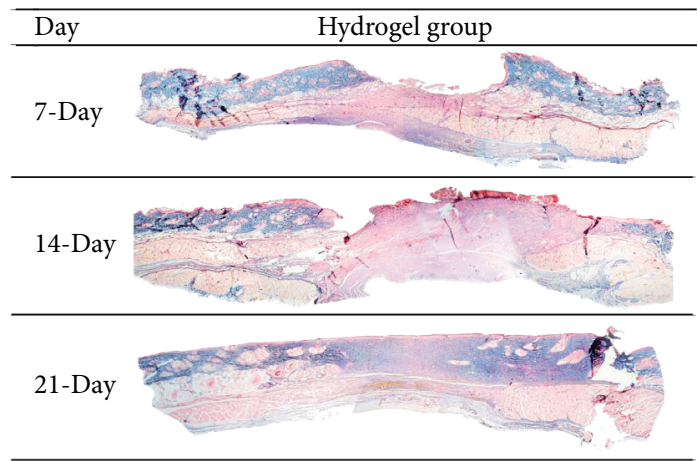

(a)

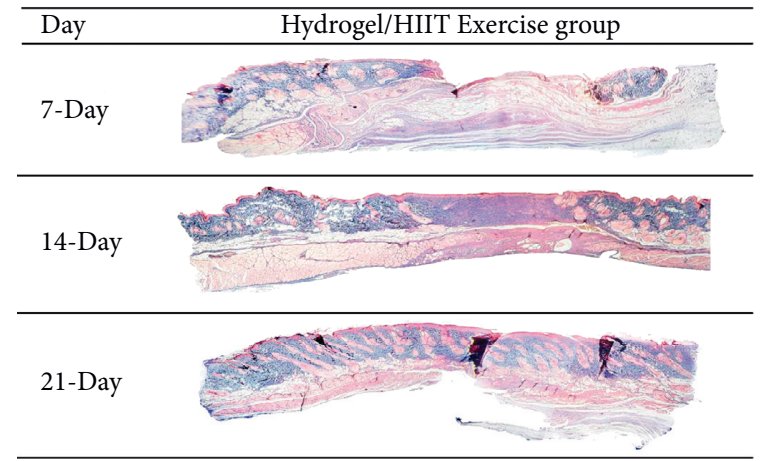

(b)

FIgURe 7: Histological analysis of hydrogel $(\mathrm{a}: \times 40)$ and hydrogel/HIIT exercise $(\mathrm{b}: \times 40)$ with Masson's trichrome staining.

considerable from margin to the center during 7 days. Furthermore, the formation of collagen substrate for wound healing was better than other treatment groups. On day 14, the rate of healing and formation of three layers were slightly suitable, and the infiltration of cells and the formation of the hypodermis were observed from the margin to the center. In addition, the formation of hair follicles and remarkable healing were detected in the margin and center of the defect, respectively. Irregular collagen substrate was considerable in the margin of the defect, and the cell infiltration and suitable healing were increased. On day 21 , the rate of healing and formation of hair follicles was considerably increased in the defect site. The formation of regular collagen clusters was observed in Masson's trichrome staining, and the hydrogel/ HIIT exercise group showed better results compared to other groups in diabetic wound healing (Figures 5(b) and 7(b)). 


\section{Discussion}

Diabetes is one of the important factors for the dysfunction of epidermal cells, fibroblasts, tissue maturation, and failed angiogenesis, which causes delayed wound healing processes. Also, diabetes is responsible for reepithelialization and wound contraction during the process of wound healing. Hydrogel wound dressings are highly regarded due to their appropriate moisturization feature for supporting cellular activities in the wound zone during the healing process. Furthermore, they provide enough flexibility, facilitate the proliferation of cells and biodegradation, and accelerate wound contraction, attachment, reepithelialization, and neovascularization. Hydrogel dressings composed of the bioactive molecule would have a significant influence on expediting the healing of the diabetic wound [18]. Collagen is also used as a therapeutic component for correcting diabetic wounds irregularities. Collagen has been known as the most significant extracellular matrix component that is essential for wound closure. Synthesis of collagen fibers, deposition, restoration, and maturation are vital phases among regeneration and repairment of tissue. Collagen synthesis is principally based on proline availability. Actively, proline is synthesized from glutamic acid in the granulation tissues, and the amount of proline synthesized by lactate increases [18]. Goldsmith et al. showed that oral administration of tadalafil once daily can be effective in the acceleration of reepithelialization and decreasing scarring in a partial thickness of animal burns model [14]. García-Villén et al. demonstrated that hydrogel of nanoclays has improved biocompatibility. Also, the hydrogel can support the motility of fibroblasts and attenuate wound healing in an in vitro study and clarify new promising prospects in novel therapeutic formulations [21]. Moreover, Soufdoost et al. reported a novel tadalafil/polycaprolactone as a novel suture that ameliorated the wound healing process by releasing the tadalafil around the sutured wound [22]. Effect of HIIT exercise in combination with resistance training on health of vessels in a one-year follow-up study of Magalhaes et al. in patients with diabetes mellitus type II has demonstrated that HIIT might be a candidate as a key method in long-term improvement of vascular complications in type II of diabetes mellitus [23].

In this study, the developed hydrogel with collagen/ nanoclay/tadalafil was prepared and investigated for wound healing in diabetic wounds with and without HIIT exercise. In vitro biocompatibility studies were performed by culturing HEK 293 cells on collagen/nanoclay/tadalafil hydrogel and the control (tissue culture plate) for 24,48 , and $72 \mathrm{~h}$. Collagen/nanoclay/tadalafil hydrogel did not exhibit any significant cytotoxicity in comparison with control. Findings revealed that the cells cultured on the collagen/ nanoclay/tadalafil hydrogel are viable and multiplying at a rate comparable to those on the control.

The antibacterial effects of skin dressing play a vital role in eliminating bacterial infections, which have been known as a major result of the wound healing postponement [24]. Mojtaba et al. used the nanoclay and chitosan to prepare an active nanocomposite according to their antimicrobial features to assess its influence on the microbial limitation. The chitosan and nanoclay nanocomposite had excellent executed and well antibacterial effects against microbial limitations (coliforms, Salmonella spp., coagulase-positive Staphylococcus, E. coli, yeast, and mold) [25]. Olivetti et al. showed that DDSA (dodecenylsuccinic anhydride)-collagen hydrogels, either unloaded or loaded with simvastatin, showed sustained antimicrobial activity against Pseudomonas aeruginosa and Staphylococcus aureus for 72 hours, maybe because of the hydrophobic DDSA chain interaction with cell wall bacteria. The antimicrobial activity was stronger against S. aureus. Also, collagen hydrogels provided a prolonged antibacterial activity when they involved simvastatin, and findings revealed that these materials could stimulate macrophages which is useful in wound healing processes [26]. The MTT assay of Chakrabarti et al. study showed that bFGF and collagen matrix hydrogel had minimal toxicity on the L929 mouse fibroblast cell line, did not inhibit cell proliferation, and could be further investigated in burn wounds [27]. In the present study, the antibacterial evaluations showed that the number reduction of $\mathrm{E}$. coli was more than S. aureus. The collagen/nanoclay/tadalafil hydrogel displayed an inhibition zone in comparison with the standard antibiotic disc. Therefore, this result revealed that the hydrogel might conduct an antibacterial effect on the healing process of the wound.

Wound contraction has been known as a progressive process that appears in the matrix of tissue and accelerates healing by providing adequate granulation tissue to restore the tissue damage. The wound healing process is crucially correlated with reepithelialization due to regenerating and attenuating the surface of the wound [18]. Huang et al. have used a clay-reinforced composite film, polycaprolactone/ chitosan/curcumin (PCL/CS/Clay/Cur) for wound dressing, and they displayed the inhibitory effect of this film of clay on bacteria $(168 \mathrm{mg} / \mathrm{ml})$. Moreover, they demonstrated that Cur-loading films with increasing clay could be high potential candidates for the caring wound [28]. Thangavel et al. developed hydrogels containing LG (L-glutamic acid) and CS (chitosan) to treat diabetic ulcers. They displayed that the LG + CS hydrogel facilitated vascularization for the acceleration of diabetic wound healing. These findings revealed that the combination of LG can ameliorate deposition of collagen, vascularization, and purposes in the acceleration of diabetic wounds regeneration [18]. Neovascularization or angiogenesis has a crucial role in the rebuilding of injured skin and avoiding subsequent complications such as nonhealing diabetic wounds. Neovascularization deficiency, especially deficit information of microvascular network, refers to the interruption of tissue repair in diabetic circumstances [18]. In this study, it was detected that collagen/ nanoclay/tadalafil hydrogel demonstrated fine adhesiveness when administered at the surface of the wound topically, and the macroscopic results demonstrated that the wound contraction was considerable in the hydrogel/HIIT exercise and hydrogel groups compared with the HIIT exercise and control groups during 21 days. The hydrogel-treated diabetic wounds displayed complete epithelialization in 21 days. The present results showed an increased rate of epithelialization 
in diabetic rats. These results display that using collagen/ nanoclay/tadalafil hydrogel with HIIT exercise helps in wound healing. The rate of wound healing in the control and treated groups showed that the rate of regeneration in the hydrogel/HIIT exercise group was higher than that in the hydrogel group in 7 days and the connective tissue formation and cell infiltration were observed. In addition, this rate was very considerable in the hydrogel/HIIT exercise and hydrogel compared to the HIIT exercise and control groups. The rate of wound healing, connective tissue formation, and cell infiltration was very significant in the HIIT exercise group compared with the control in 7 days. The rate of wound healing, infiltration of cell, and hair follicle formation in the hydrogel/HIIT exercise was significant compared to the hydrogel and HIIT exercise group in 14 days. Also, treated groups with HIIT exercise can play a crucial role in the amelioration of wounds compared to the control group. Thus, wound healing, the formation of a regular layer of collagen, hair follicles, and the formation of three layers of wounds were accelerated and better performed in the hydrogel/HIIT exercise and hydrogel groups than those in the control and HIIT exercise groups, but hydrogel/HIIT exercise was remarkably more successful than the hydrogel group in wound repairing, forming skin layers and classifying arranged collagen. Enhanced content of collagen is detected in the hydrogel/HIIT exercise and hydrogel groups. Therefore, increment of collagen content is considered in the granulation tissues. Assessment of Masson's trichrome staining revealed that collagen/nanoclay/tadalafil hydrogel treatment enhanced the content of the collagen.

Tan et al. investigated the properties of a novel composite biomaterial, a collagen scaffold loaded with CBD-VEGF, for healing of the wound in a diabetic rat model. They found that collagen scaffold loaded with CBD-VEGF facilitated healing in the diabetic wound of the rat model, which may have promising therapeutic approaches for diabetic wound treatment [29]. Long et al. provided a collagen membrane as a drug delivery scaffold to assess whether the combined use of VEGF and SDF- $1 \alpha$ has a synergistic effect on the treatment of diabetic wound healing. Their long-term findings elucidate that the comodified scaffold can accelerate wound healing, facilitate regeneration of blood vessels, and promote proliferation of assist cells, accumulation of extracellular matrix, and reepithelialization. Altogether, the study demonstrated that the CBD-SDF- $1 \alpha$ and CBD-VEGF comodified scaffold support rapid recovery from diabetic wounds through matching inflammation and angiogenesis [30].

In this study, the amount of collagen in the HIIT exercise group was increased after 21 days, while this amount was much higher in the hydrogel/HIIT exercise and hydrogel groups than that in the control and HIIT exercise groups. Also, the presence of fibroblasts in the hydrogel/HIIT exercise and hydrogel groups was higher than that in the HIIT exercise and control groups. Moreover, this amount of fibroblasts was significant in the hydrogel/HIIT exercise group compared to the hydrogel. Epidermis density was higher in the hydrogel/HIIT exercise group than that in the hydrogel group, and the hydrogel/HIIT exercise and hydrogel groups were significantly different from the control and HIIT exercise groups. The rate of vascularization was decreased in the HIIT exercise group during 21 days that it was significantly different from the control group, and it indicated that the HIIT exercise could accelerate the healing by increasing vascularization on days 7 and 14, while the rate of vascularization was increased in the control group on day 21. The increase of vascularization rate showed the acceleration of healing in the hydrogel/HIIT exercise and hydrogel groups on days 7 and 14. After 21 days, the number of vessels was decreased, which indicated a suitable process of regeneration. The number of hair follicles was visible only on day 21 in the control group, and the number of hair follicles in the two groups of hydrogel/HIIT exercise and hydrogel was higher than that in the control and HIIT exercise groups with a significant difference. The presence of inflammatory cells in the control and HIIT exercise groups showed a significant difference between days 7 and 14 compared to the hydrogel/ HIIT exercise and hydrogel groups, and inflammatory cells were decreased after day 21 . The hydrogel/HIIT exercise and hydrogel groups showed a significant reduction of inflammatory cell infiltration on days 7 and 14, and this amount was reduced on day 21. Generally, the amount of inflammatory in the hydrogel/HIIT exercise and hydrogel groups was remarkably different from the control and HIIT exercise groups.

\section{Conclusion}

In conclusion, collagen/nanoclay/tadalafil hydrogel facilitated movements of cells at the wound zone for improving scar formation and accelerating wound contraction. The HIIT exercise with hydrogels decreased the duration of epithelialization, increased wound contraction rate, and enhanced crosslinking and the content of collagen in diabetic animal models. These findings have also revealed that hydrogel-involved collagen/nanoclay/tadalafil can be conducted as a high potential therapeutic approach in the amelioration of diabetic wounds. Therefore, hydrogel/HIIT exercise hydrogel would be an appropriate candidate for wound healing due to the acceleration of wound healing, the formation of hair follicles, and the arrangement of collagen fibers.

\section{Data Availability}

All the data generated or analyzed during this study are included in this published article, and the datasets analyzed to support the findings of this study are available from the corresponding author upon request.

\section{Ethical Approval}

All of the in vitro and in vivo tests were approved by the Ethical Committee for Animal Research, Tehran, Iran.

\section{Conflicts of Interest}

The authors declare that there are no conflicts of interest regarding the publication of the present paper. 


\section{References}

[1] S. Patel, S. Srivastava, M. R. Singh, and D. Singh, "Mechanistic insight into diabetic wounds: pathogenesis, molecular targets and treatment strategies to pace wound healing," Biomedicine \& Pharmacotherapy, vol. 112, Article ID 108615, 2019.

[2] S. M. Ayuk, H. Abrahamse, and N. N. Houreld, "The role of matrix metalloproteinases in diabetic wound healing in relation to photobiomodulation," Journal of Diabetes Research, vol. 2016, Article ID 2897656, 2016.

[3] K. C. Broussard and J. G. Powers, "Wound dressings: selecting the most appropriate type," American Journal of Clinical Dermatology, vol. 14, no. 6, pp. 449-459, 2013.

[4] S. A. Hosseini, O. R. Slehi, F. Keikhosravi et al., "Mental health benefits of exercise and genistein in elderly rats," Experimental Aging Research, pp. 1-16, 2021.

[5] S. Ito, "High-intensity interval training for health benefits and care of cardiac diseases-the key to an efficient exercise protocol," World Journal of Cardiology, vol. 11, no. 7, pp. 171-188, 2019.

[6] F. Torma, Z. Gombos, M. Jokai, M. Takeda, T. Mimura, and Z. Radak, "High intensity interval training and molecular adaptive response of skeletal muscle," Sports Medicine and Health Science, vol. 1, no. 1, pp. 24-32, 2019.

[7] K. Norton, L. Norton, and D. Sadgrove, "Position statement on physical activity and exercise intensity terminology," Journal of Science and Medicine in Sport, vol. 13, no. 5, pp. 496-502, 2010.

[8] J. S. Ramos, L. C. Dalleck, A. E. Tjonna, K. S. Beetham, and J. S. Coombes, "The impact of high-intensity interval training versus moderate-intensity continuous training on vascular function: a systematic review and meta-analysis," Sports Medicine, vol. 45, no. 5, pp. 679-692, 2015.

[9] A. Mitropoulos, A. Gumber, H. Crank, M. Akil, and M. Klonizakis, "Exploring the feasibility of an exercise programme including aerobic and resistance training in people with limited cutaneous systemic sclerosis," Clinical Rheumatology, vol. 39, no. 6, pp. 1889-1898, 2020.

[10] D. Baltzis, I. Eleftheriadou, and A. Veves, "Pathogenesis and treatment of impaired wound healing in diabetes mellitus: new insights," Advances in Therapy, vol. 31, no. 8, pp. 817-836, 2014.

[11] G. M. Virador, L. de Marcos, and V. M. Virador, "Skin wound healing: refractory wounds and novel solutions," Methods in Molecular Biology (Clifton, N.J.), vol. 1879, pp. 221-241, 2019.

[12] A. Jarad, A. Hamoody, M. Hasan et al., "Diabetic wound healing enhancement by tadalafil," International Journal of Pharmaceutical Research, vol. 12, pp. 841-847, 2020.

[13] C. Davenport and A. Dubin, "Tadalafil therapy and severe chronic foot wound resolution," International Wound Journal, vol. 12, no. 6, pp. 733-736, 2015.

[14] K. Goldsmith, E. Goradia, S. A. McClain, S. Sandoval, and A. J. Singer, "The effect of tadalafil on reepithelialization and scarring of partial thickness porcine burns," Wound Repair and Regeneration, vol. 28, no. 1, pp. 26-32, 2020.

[15] K. T. Huang, Y. L. Fang, P. S. Hsieh, C. C. Li, N. T. Dai, and C. J. Huang, "Non-sticky and antimicrobial zwitterionic nanocomposite dressings for infected chronic wounds," Biomaterials Science, vol. 5, no. 6, pp. 1072-1081, 2017.

[16] K. T. Huang, Y. L. Fang, P. S. Hsieh, C. C. Li, N. T. Dai, and C. J. Huang, "Zwitterionic nanocomposite hydrogels as effective wound dressings," Journal of Materials Chemistry B, vol. 4, no. 23, pp. 4206-4215, 2016.
[17] A. D. Metcalfe and M. W. J. Ferguson, "Tissue engineering of replacement skin: the crossroads of biomaterials, wound healing, embryonic development, stem cells and regeneration," Journal of The Royal Society Interface, vol. 4, no. 14, pp. 413-437, 2007.

[18] P. Thangavel, B. Ramachandran, S. Chakraborty, R. Kannan, S. Lonchin, and V. Muthuvijayan, "Accelerated healing of diabetic wounds treated with L-glutamic acid loaded hydrogels through enhanced collagen deposition and angiogenesis: an in vivo study," Scientific Reports, vol. 7, no. 1, Article ID 10701, 2017.

[19] E. Robinson, C. Durrer, S. Simtchouk et al., "Short-term highintensity interval and moderate-intensity continuous training reduce leukocyte TLR4 in inactive adults at elevated risk of type 2 diabetes," Journal of Applied Physiology (1985), vol. 119, no. 5, pp. 508-516, 2015.

[20] M. V. Machado, A. B. Vieira, F. G. da Conceição, A. R. Nascimento, A. C. L. da Nóbrega, and E. Tibirica, "Exercise training dose differentially alters muscle and heart capillary density and metabolic functions in an obese rat with metabolic syndrome," Experimental Physiology, vol. 102, no. 12, pp. 1716-1728, 2017.

[21] F. García-Villén, A. Faccendini, D. Miele et al., "Wound healing activity of nanoclay/spring water hydrogels," Pharmaceutics, vol. 12, no. 5, p. 467, 2020.

[22] R. S. Soufdoost, S. A. Mosaddad, Y. Salari et al., "Surgical suture assembled with tadalafil/polycaprolactone drug-delivery for vascular stimulation around wound: validated in a preclinical model," Biointerface Res Appl Chem, vol. 10, pp. 6317-6327, 2020.

[23] J. P. Magalhães, X. Melo, I. R. Correia et al., "Effects of combined training with different intensities on vascular health in patients with type 2 diabetes: a 1-year randomized controlled trial," Cardiovascular Diabetology, vol. 18, no. 1, p. 34, 2019.

[24] M. Bahadoran, A. Shamloo, and Y. D. Nokoorani, "Development of a polyvinyl alcohol/sodium alginate hydrogelbased scaffold incorporating bFGF-encapsulated microspheres for accelerated wound healing," Scientific Reports, vol. 10, no. 1, p. 7342, 2020.

[25] M. V. Mojtaba, H. Seyed Masoud, M. Ali, J. Mahdi, and M. Hadi, "Investigation of the antimicrobial properties of nanoclay and chitosan based nanocomposite on the microbial characteristics of Gouda cheese," Iranian Journal of Microbiology, vol. 12, no. 2, 2020.

[26] C. E. Olivetti, M. I. Alvarez Echazú, O. Perna et al., "Dodecenylsuccinic anhydride modified collagen hydrogels loaded with simvastatin as skin wound dressings," Journal of Biomedical Materials Research Part A, vol. 107, no. 9, pp. 1999-2012, 2019.

[27] S. Chakrabarti, B. Mazumder, J. Rajkonwar, M. P. Pathak, P. Patowary, and P. Chattopadhyay, "bFGF and collagen matrix hydrogel attenuates burn wound inflammation through activation of ERK and TRK pathway," Scientific Reports, vol. 11, no. 1, p. 3357, 2021.

[28] Y. Huang, N. Dan, W. Dan, and W. Zhao, "Reinforcement of polycaprolactone/chitosan with nanoclay and controlled release of curcumin for wound dressing," ACS Omega, vol. 4, no. 27, pp. 22292-22301, 2019.

[29] Q. Tan, B. Chen, X. Yan et al., "Promotion of diabetic wound healing by collagen scaffold with collagen-binding vascular endothelial growth factor in a diabetic rat model," Journal of Tissue Engineering and Regenerative Medicine, vol. 8, no. 3, pp. 195-201, 2014.

[30] G. Long, D. Liu, X. He et al., "A dual functional collagen scaffold coordinates angiogenesis and inflammation for diabetic wound healing," Biomaterials Science, vol. 8, no. 22, pp. 6337-6349, 2020. 